\title{
Small molecule drug development for rare genodermatoses - evaluation of the current status in epidermolysis bullosa
}

\author{
Verena Wally ${ }^{1 *} \mathbb{D}$, Manuela Reisenberger ${ }^{2}$, Sophie Kitzmüller ${ }^{1}$ and Martin Laimer ${ }^{1,2}$
}

\begin{abstract}
Background: Hereditary epidermolysis bullosa (EB) comprises a heterogeneous group of rare genodermatoses, which are caused by mutations in genes involved in the maintenance of the structural and functional integrity of dermo-epidermal adhesion in various stratified epithelia. In severe variants, generalized skin disease, extracutaneous manifestations and multi-organ involvement cause considerable morbidity and mortality. Causal and early treatment by re-expression of a respective mutated gene is the major long-term goal in therapy development. However, characterization and targeted modulation of pathogenic molecular cascades in EB also holds great promise as a symptom-relieving approach to ameliorate phenotype, complications and quality of life. Small molecules are chemical structures of less than $900 \mathrm{Da}$ that can diffuse across cell membranes and interfere with target biomolecules, thus influencing their function at different levels. They constitute the vast majority of active components of all approved drugs.
\end{abstract}

Methods: We performed PubMed and Google Scholar search for publications and screened FDA- and EMA-hosted clinical trial registries to identify studies using small molecule-based drugs for epidermolysis bullosa. Upon detailed analysis this resulted in the identification of a total of 84 studies.

Results: We identified 52 publications and 32 registered trials that investigate small molecules for their safety and efficacy as treatment for different aspects of epidermolysis bullosa. Further, a total of 38 different small molecules clinically used in EB were found. Most frequent outcome measures concerned wound healing, reduction in blister numbers, as well as reduction of itch and pain, predominantly for EBS and RDEB.

Conclusion: We provide a comprehensive summary of the current status of clinical small molecule development for EB and discuss prospects and limitations in orphan drug development for rare conditions like EB.

Keywords: Epidermolysis bullosa, Genodermatosis, Small molecules, Orphan drugs, Clinical trial, Case study

\footnotetext{
* Correspondence: v.wally@salk.at

${ }^{1}$ EB House Austria, Research Program for Molecular Therapy of

Genodermatoses, Department of Dermatology and Allergology, University

Hospital of the Paracelsus Medical University, 5020 Salzburg, Austria

Full list of author information is available at the end of the article
}

(c) The Author(s). 2020 Open Access This article is licensed under a Creative Commons Attribution 4.0 International License, which permits use, sharing, adaptation, distribution and reproduction in any medium or format, as long as you give appropriate credit to the original author(s) and the source, provide a link to the Creative Commons licence, and indicate if changes were made. The images or other third party material in this article are included in the article's Creative Commons licence, unless indicated otherwise in a credit line to the material. If material is not included in the article's Creative Commons licence and your intended use is not permitted by statutory regulation or exceeds the permitted use, you will need to obtain permission directly from the copyright holder. To view a copy of this licence, visit http://creativecommons.org/licenses/by/4.0/ The Creative Commons Public Domain Dedication waiver (http://creativecommons.org/publicdomain/zero/1.0/) applies to the data made available in this article, unless otherwise stated in a credit line to the data. 


\section{Introduction}

Recent advances in understanding pathomechanisms of different epidermolysis bullosa (EB) subtypes have facilitated the development of novel, targeted therapeutics. These approaches address debilitating symptoms of EB, like recurring or chronic wounding, pain, pruritus, failure to thrive and carcinogenesis. We herein summarize latest advances in repurposing or development of small molecule-based treatments targeting pathogenic traits in $\mathrm{EB}$, while the use of biologics, as well as gene- and celltherapeutic approaches will not be discussed.

\section{The heterogeneity of EB-symptoms and complications}

The term EB comprises a group of rare, clinically and genetically heterogeneous genodermatoses characterized by moderate to excessive fragility of epithelial tissues and prototypic blistering following minimal trauma. EB is caused by mutations in genes encoding components important for the structural and functional integrity of the basement membrane zone in skin and mucous membranes $[1,2]$. Genotypic heterogeneity as well as epigenetic, environmental and socio-economic factors contribute to the broad clinical spectrum and complex genotypephenotype correlations [1].

The expression of distinct EB-associated genes in diverse epithelialized or mesenchymal organs explains extracutaneous involvement. The latter is particularly prominent in the severe subvariants, rendering EB a multi-system disease with significant morbidity and mortality due to relevant complications such as malnutrition, infections, organ failure and skin cancer [1].

Chronic tissue damage with induction and dysregulation of inflammatory pathways is a common pathogenic mechanism in EB as a mutation-based barrier disorder, although inter- and intra-individual variability of immune responses remains to be determined [3-7].

While in localized EB variants inflammatory aberrations mainly affect the micromilieu of lesional skin (leading to tissue remodeling [4-7]), a systemic impact was shown in severe subtypes such as RDEB, in which extensive cutaneous involvement is associated with a systemic inflammatory response and chronification of inflammatory cascades that contribute to the systemic morbidity of EB [8-12]. Against this background, the induction of inflammatory traits defines new therapeutic targets including skin barrier restoration, infection control/surveillance, immune response/modulation, anti-neoplastic interference and interference with epigenetic drivers of the disease. In this context, small molecule drugs provide clinical perspectives to mitigate the phenotype as well as complications.

\section{The potential of targeted small molecule drugs}

Based on the increasing knowledge on EB subtypespecific pathomechanisms, first candidates have reached clinical development, and first marketing approvals are awaited in the near future. Drugs that tackle unspecific disease symptoms like pruritus and pain have been used in EB for a long time. However, the difference to currently developed drugs is their direct targeting of defined and known EB-subtype-related targets. This renders them more specific and potentially more efficient and safe $[13,14]$.

Small molecules can interfere with a plethora of biomolecules, and are mostly designed to impair or alter target protein function. Approaches to identify candidates for drug repurposing are either rational and literature-based, or rely on the concomitant evaluation of hundreds of small molecules in high throughput screening (HTS) settings. For HTS, the design of a highly reliable, reproducible and biologically relevant assay with a representative and simple phenotype- or target-based read-out is a pre-requisite for successful HTS (Fig. 1a,c-f).

More recent developments have led to the integration of publicly available big data (e.g. transcriptomes) as an attractive source for drug development/repurposing and the advances of in silico 3D-modelling [13]. Used algorithms have reached a very high complexity and can integrate a huge amount of data, so that these platforms have the potential to translate into meaningful lead candidate nomination (Fig. 1b-f).

The classical pathway of further drug development comprises pre-clinical studies including e.g. toxicity and potency studies, formulation, pharmacokinetics. Clinical development comprises study phases I to III, with a final registration study. In the case of repurposing, single phases may be skipped under certain circumstances, which is an advantage that lines up with a reduced risk of failure due to already existing safety data, the subsequent reduced time of development, and potentially reduced development costs.

\section{Methods}

From September 2019 to April 2020, we conducted a literature review using the PubMed and Google Scholar databases. Search terms included combinations of "epidermolysis bullosa", "small molecules", "clinical trial", "case study", and "drugs". In addition, the clinical trial databases of the FDA [15] and EMA [16] were searched for trials for epidermolysis bullosa. Publications and trials investigating drugs in a clinical setting were included, those investigating biologics, cell- or gene-therapeutic approaches were excluded. In addition, non-Englishlanguage publications, review articles and pre-clinical studies were excluded. Following these inclusion and exclusion criteria, a total of 52 publications and 32 registered clinical trials were selected for evaluation in this review (Suppl. Figure 1). 


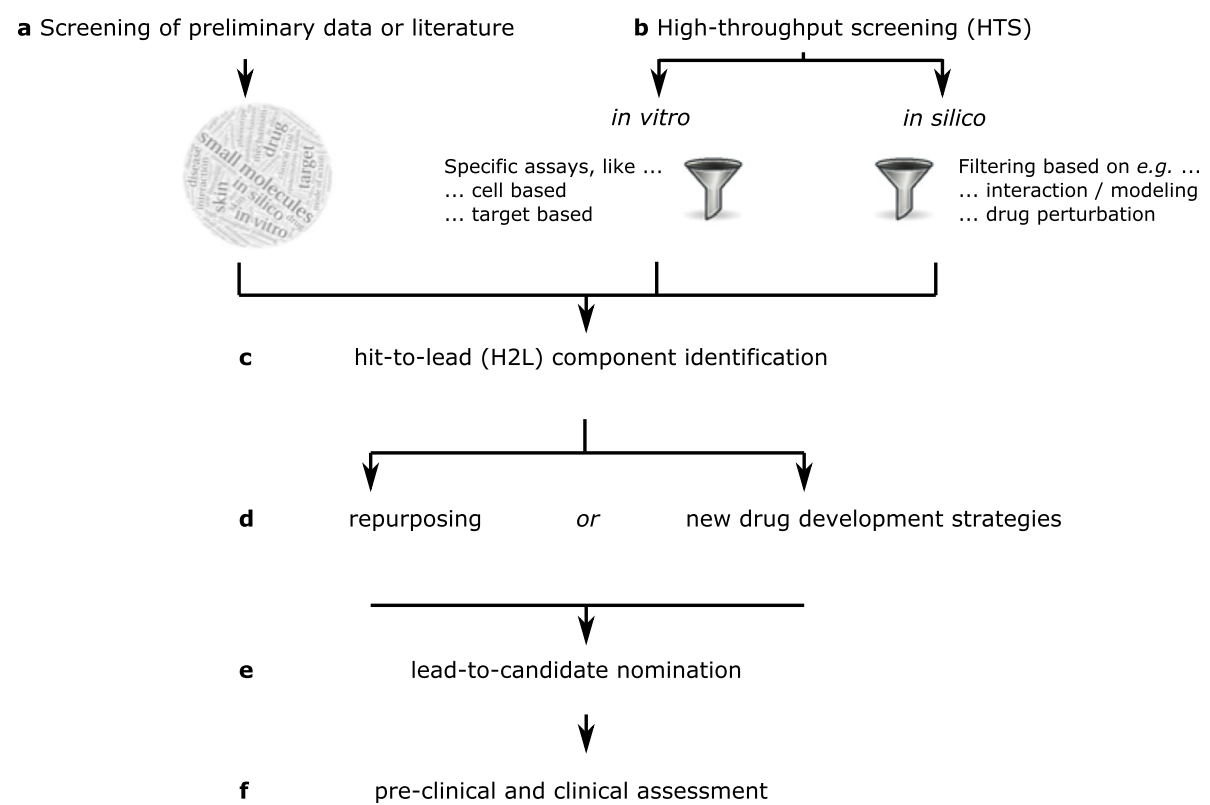

Fig. 1 Simplified scheme of drug development options. a While screening of preliminary data or literature restricts number of hits (candidates that potentially interfere with a predefined target or pathway) to such already published or rationally identified, b high-throughput screening (HTS) can screen thousands of compounds simultaneously. The latter can be done in vitro using predefined assays with clear read-outs in combination with drug libraries, as well as in silico based on big data to identify drug-drug or drug-disease similarities, or using bioinformatic modeling. c Both approaches result in a number of hits, out of which lead candidates for further development are selected upon further confirmatory testing. $\mathbf{d}$ For lead components, further predefined testings are performed, which are amongst others dependent on whether a drug is already approved for other conditions or if the whole drug development process has to be performed. e Resulting candidates can then be taken forward to pre-clinical testings first, and if passing all exigencies, to clinical assessment (f)

\section{Results}

Publications describing first attempts of drug repurposing for EB date back to the 80s, and have been used even long before ([17-54] Additional file 2). The following section gives an overview of the latest publications (2015-) on (randomized)-controlled trials ((R)CT) and currently registered active clinical studies (Additional file 3) in which small molecules are investigated for their potential utility in EB. Small molecule drugs that are currently being developed at a pre-clinical stage will not be discussed. Overall, publications on 28 different small molecules clinically used in EB were found, and 20 in registered clinical trials, with an overlap of 10 drugs.

\section{Summary of publications on recent (R)CT-trials and current clinical development status}

Gentamicin is an aminoglycoside antibiotic, which has been applied in several genetic disorders such as cystic fibrosis [55] and hemophilia [56]. Aminoglycosides promote stop codon read-through by enabling the ribosome to pass the stop codon in the mRNA resulting in restored protein translation [57]. In a RCT trial, five RDEB patients with nonsense mutations in the COL7A1 gene were treated with either topically applied or locally injected gentamicin, which induced anchoring fibril formation, improved wound closure and reduced blister numbers [58]. Further studies using topical formulations in JEB reported improved wound healing and reduction in blisters numbers $[59,60]$. In a retrospective study including five JEB patients, the systemic application of gentamicin was assessed for the first time. Gentamicin was applied intravenously (i.v.) followed by intramuscular (i.m.) injection, or i.m. only, leading to improved skin stability and quality-of-life (QoL) in 4/5 patients. However, in this study it did not postpone lethality [61]. Currently, two trials involving gentamicin treatment are running (NCT04140786, NCT03526159), aiming to increase laminin-332 and C7 expression in order to induce the generation of new hemidesmosomes and anchoring fibrils, respectively, monitored by immune- and electron microscopy of skin biopsies. Topical and intravenous applications are compared.

Betulin: Betulin-enriched triterpene extract is mainly isolated from birch bark and is already approved and used for partial thickness wounds. A semisolid gel formulation was used in a phase II, reference therapycontrolled trial, in which long-standing and acute RDEB-wounds were topically treated and analyzed for enhanced re-epithelialization [62]. Betulin mainly effects the inflammatory phase of the wound healing process, where it positively modulates mediators of inflammation, like COX-2 or IL-6 [63]. In addition, it is also thought to 
have anti-cancer effects [64, 65]. While it was shown that the formulation was well tolerated, only a slight, statistically not significant effect on wound healing was observed. However, results encouraged a larger clinical trial that is currently ongoing (NCT03453632), where betulin is tested as topical formulation for its efficacy and safety in EB. Results of verum and placebo groups will be compared by evaluating answers to the "Foot Health Status Questionnaire".

Diacerein: For the sev-EBS, which is caused by dominantly inherited mutations within either KRT5 or KRT14, it was shown that a pro-inflammatory signaling-cascade triggered by aggregation of mutated keratins is linked to skin fragility. In a positive feedback loop, IL-1ß is activated and released from effected keratinocytes and, besides paracrine signaling, enhances the expression of KRT14, resulting in a perpetuum mobile-like state [66]. Diacerein, a small molecule derived from the rhubarb root, can interfere with the IL-1ß mediated inflammatory pathway at several levels [67-73]. In a phase II/III RCT that was based on a previous pilot study [74], diacerein was shown to significantly reduce blister numbers in sevEBS patients in a 4-week treatment episode. This effect further increased to the end of a 3-months follow-up [75]. After two investigator-driven trials, further studies using a $1 \%$ Diacerein-containing ointment were conducted. While one study was prematurely terminated, a further trial is ongoing which focuses on pharmacokinetics after long-term use (NCT03389308).

Serlopitant: In EB patients, itch is a major distressing symptom that worsens the disease phenotype due to scratching-induced wounding. The neurokinin-1 (NK1) receptor plays a major role in the pathogenesis of itch, and NK1-receptor antagonists, such as Serlopitant, are used to disrupt itch signaling [76]. In a RCT with 14 EBpatients, oral serlopitant led to itch reduction, although results were not statistically significant [77]. Currently, a larger clinical trial is ongoing with the aim to achieve $\mathrm{a} \geq 3$-points reduction in itch severity after 2 months of treatment (NCT03836001).

Epigallocatechin-3-gallate (EGCG): Matrixmetalloproteinase 7 (MMP7) is expressed at increased levels in the skin of RDEB patients, where it contributes to the degradation of $C 7$ [78]. Therefore, the MMP7 inhibitor EGCG was used to treat 17 RDEB patients in an RCT-clinical trial with the aim to reduce blister numbers as well as itch, and to improve wound healing. Although a reduction in blister numbers was observed in $50 \%$ of patients compared to placebo, statistical analysis showed no significant difference [79]. Currently, no further trials are registered.

\section{Further small molecule drugs in currently ongoing clinical} trials

Coenzyme Q10: In an open label trial (NCT02793960), a Coenzyme Q10 (ubidecarenone) cream is tested for its effect on wound healing upon application on at least one target wound and unaffected skin. The respective mode of action is to target mitochondria, e.g. via regulation of the cell metabolism during remodeling and expression of structural proteins, in order to strengthen the skin. Safety is controlled by blood tests, efficacy by changes in visual analog scale (VAS) pain scale and EBDASI questionnaires [80].

Botulinum toxin is frequently used for the treatment of wrinkles and hyperhidrosis, however, in the context of EB, Swartling and Holahan et al. reported on treating plantar blistering and pain in EBS patients. Intradermal injections led to a reduction of pain and reduced blister numbers, lasting for $<4$ months [81, 82]. In a second case report, botulinum toxin was injected into the internal anal sphincter of an RDEB-patient over a 2-year period leading to relieved spasm and pain due to anal blistering, with a sustained effect lasting 4 years post treatment [83]. Following these studies, a currently recruiting intra-patient controlled trial (NCT3453632) evaluates the effect of botulinum toxin in a bigger patient cohort. The drug is injected into the sole of one foot, the other foot receives placebo. The effect in reduction of blister numbers is measured using an "Improvement Global Assessment" (IGA) score.

Pregabalin: This approved anticonvulsant is investigated to ameliorate pain and itch in RDEB patients. Via the inhibition of calcium currents, pregabalin reduces the release of neurotransmitters like glutamate, norepinephrine and substance-P in the central nervous system, and thus lowers neuronal excitability [84]. The systemic treatment is administered orally. The double-blinded cross over design allows the patients to serve as their own control. Primary outcome measures are patient reported pain scores (NCT03928093).

Sirolimus: Sirolimus is an mTOR inhibitor known for its anti-inflammatory effects [85]. In EB it is used to downregulate the translation of defective keratin proteins, therefore leading to an improvement of plantar skin lesions. Data are collected from a pedometer and lesion measurements with 3D-photography (NCT02960997).

Rigosertib: Squamous cell carcinomas (SCC), one of the most threatening co-pathologies in RDEB, is treated with Rigosertib, a polo-like kinase (PLK)-1 inhibitor leading to apoptosis in cancer cells [86]. Oral and i.v. applications are available. Tumor progression is determined via RECI ST (response evaluation criteria in solid tumors) evaluation and biomarker analysis (NCT03786237).

Ropivacaine: Ropivacaine, a local anesthetic, reduces pain via impulse conduction blockage through inhibition 
of the sodium ion influx in nerve cells [87]. In this trial (NCT03730584) it is applied on painful wounds before dressing change. Patient or caregiver indicates pain levels during the procedure using respective analogue scale.

In summary, while most published clinical studies used oral administration (oral: 30/52; topical: 16/52, others: 8/ $52)$, in most currently registered trials drugs are applied topically (21/32, oral: $9 / 32)$. This may reflect the striving to develop drugs that can be administered by the patient himself and at the same time to avoid side effects upon oral administration.

When analyzing outcome measures we found that nearly all studies could be assigned to either of 5 groups: a) improvement of wound healing, b) reduction of blister numbers, c) reduction of itch and d) pain, and e) the prevention or treatment of SCC. Here, the most striking differences are evident in the proportion of the endpoints wound closure versus reduction of blister numbers (published: 21\% / 56\%; registered: 75\% / 25\%).

Finally, differences in EB subtypes included differs between publications and registered trials (registered: 15/ 32 open for all EB subtypes, whereas 49/52 published trials included only distinct subvariants), pointing towards the aim of industry to generate sufficient evidence for benefit as well as broad patient applicability of innovative therapeutics, whereas investigator-driven trials tend to focus on very specific and individual symptoms (Fig. 2, Table 1).

The positive outcome of several studies in reducing secondary disease manifestations will encourage the use of these drugs in the future. Some medications are already in advanced-phase trials and might enter the market within the next decade. The availability of symptom-relieving drugs that have been systematically tested for EB patients will likewise offer new treatment modalities, such as stand-alone treatments for milder subvariants or distinct complications, as well as combination regimes with other drugs/biologics or causal treatments approaches like gene-therapy. In this context, a consensus in the EB expert community on the complementarity of different therapeutic approaches to optimize treatment outcomes was recently reported, involving e.g. pain-relieving drugs such as opioids with non-pharmacological interventions $[88,89]$.

\section{Discussion}

Currently, no general curative therapy is available for EB. In light of the morbidity and lethality of numerous EB-subvariants, causative therapeutic approaches, like gene-, protein-, and cell-based therapies, are urgently awaited. While treatments like cDNA replacement and cell-therapy have entered clinical development, the availability of such treatments for all patients with EB is not

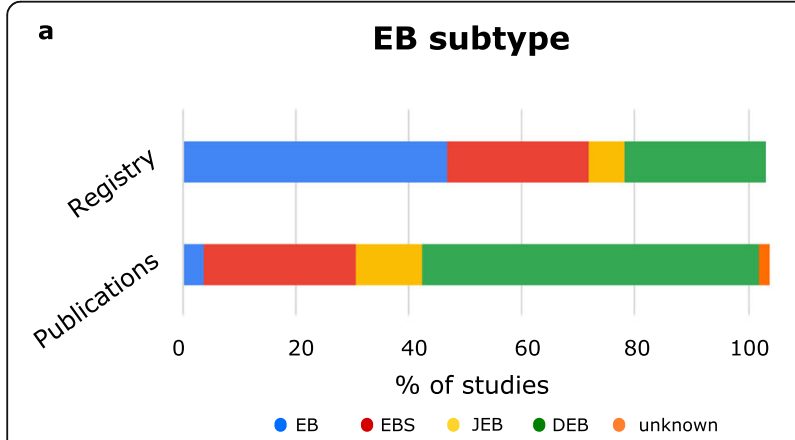

b

Route of administration
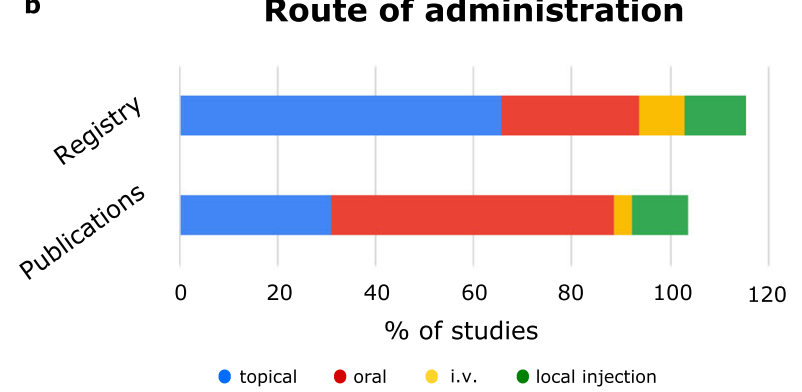

c

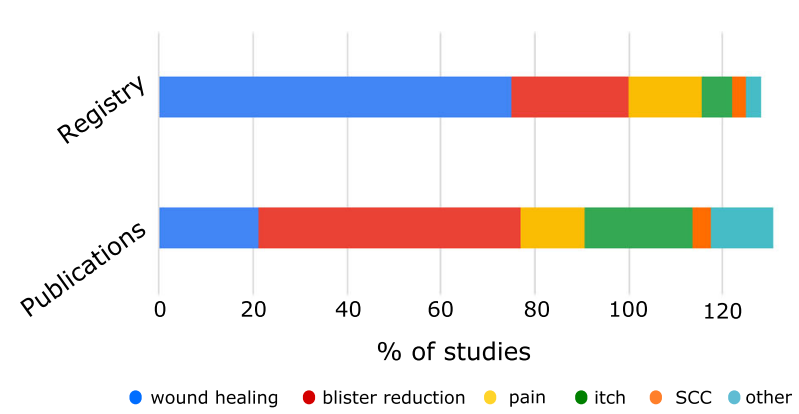

Fig. 2 Summary of distribution of EB subtypes, outcome measures and routes of administration in reviewed trials. Percentage of respective parameters (a. EB subtype. b. outcome measure, c. route of administration) is given. A total of $>100 \%$ derives from the fact, that some studies have multiple specifications per criteria (e.g. outcome measure: wound healing and reduction of pain) 
Table 1 Table giving the absolute numbers of studies investigating selected criteria (EB subtype, route of administration, outcome measure). As some studies list two possibilities for distinct criteria, respective sums might exceed the total of studies

\begin{tabular}{lll}
\hline & Publication $(\boldsymbol{n}=52)$ & Database $(\boldsymbol{n}=32)$ \\
\hline Outcome measure & 11 & 24 \\
Wound healing & 29 & 8 \\
Blister reduction & 7 & 5 \\
Pain & 12 & 2 \\
Itch & 2 & 1 \\
SCC & 7 & 1 \\
Other & & \\
Route of administration & & 21 \\
Topical & 16 & 9 \\
Oral & 30 & 3 \\
i.v. & 2 & 4 \\
Local injection & 6 & 0 \\
Unknown & 2 & \\
EB Subtype & & 15 \\
EB & 2 & 8 \\
EBS & 14 & 0 \\
JEB & 6 & 2 \\
DEB & 31 & \\
unknown & 1 & \\
\hline
\end{tabular}

yet in reach due to limitations in terms of feasibility, efficacy, specificity, and safety [90-93]. Against this background, investigating new or otherwise used drugs for their effectiveness in EB gives promising perspectives for treatment approaches that positively impact symptoms, increase QoL and prevent/treat severe complications.

Generated evidence on efficacy of small molecules from published clinical trials is generally limited, reflecting mostly intrinsic and methodological constraints in small populations, including sample size requirements, recruitment failures, restricted replication and statistical power, or shortcomings in determining clinically meaningful endpoints and valid outcome measures. When analyzing publications on RCT-trials for EB $(n=11)$, less than $50 \%$ reached statistically significant efficacy, even though all but one trial report a positive effect of the treatment. This is most likely due to that fact that recruitment requirements were only met in a minority of trials, although sample size calculations were done for $5 / 11$ trials. Difficulties in patient recruitment were mentioned by nearly all authors, even though substances were well tolerated with favorable safety profile, and without reported severe adverse events. Likewise, sample size issues and failures to reach statistical significance may underline the fact that almost $10 \%$ of pharma-driven trials had to be prematurely terminated.

As awareness towards rare diseases is raising, specific programs and adaptions of guidelines for respective drug developments are established to address deficits and promote accessibility to innovative therapeutics for EB patients. These include new methodological strategies [94], fast track designations, or the possibility to receive an orphan drug status by agencies like FDA or EMA. The latter gives the holder certain advantages, like the creation of financial incentives to develop those drugs. These incentives are being increasingly perceived and by now $7 / 38$ here discussed compounds ( +1 not registered or published) have been designated as orphan drugs. Two (diacerein, betulin) have additionally received a fast-track designation.

In terms of patient-centricity of clinical research as key measure to optimize trail recruiting and adherence, most selected endpoints of the analyzed published studies reflect treatment needs that are reported to be as much patient-relevant as currently unmet [95]. In particular, these include itch (12/52), pain (7/52), wound closure (11/52) and blister reduction (29/52). The numbers in registered clinical trials are diverging, where 24/ 32 focus on wound healing, and eight on reduction of blister numbers (Fig. 2a). This may reflect the fact that while wound healing is a generally well accepted endpoint, no guidelines for analyzing blister numbers exist. This, however, may be a hurdle for the development of drugs that address the pathophysiology of blisters, which is different to chronic wounds.

In general, perspectives for symptomatic immunomodulation in EB (including the evaluation of agents approved/tested for other immunologically mediated diseases like atopic dermatitis) are promising. However, it remains to be determined to which extent individual immune profiling is necessary to translate repurposing approaches into an efficient, safe, feasible, and tolerable therapeutic rational. Most probably, a multistep targeting approach and combinatory regimens will be essential for sustained efficacy in EB. In addition, approaches may further focus on subtypes for which the risk-benefitratio for current state of the art gene therapies are not yet beneficial (e.g. EBS), as well as on those associated with particularly high burden and numerous complications (e.g. DEB), which is reflected in the studies here analyzed. Likewise, the high proportion of topically and orally administered drugs (used in $\sim 90 \%$ of all trials) reflects the effort to increase feasibility and tolerability for the vulnerable EB cohort.

\section{Conclusion}

In summary, recent clinical trials are based on increasing understanding of pathomechanisms, resulting in more 
targeted approaches with clearly defined mechanisms of action of the investigational product. This renders the probability to conduct successful clinical trials more likely, and increases the chance for further clinical development until marketing approval. While new drug entities will still be coming up for diverse diseases and also for $\mathrm{EB}$, drug repurposing holds great potential and is foreseen a promising, additional route to go.

\section{Supplementary information}

Supplementary information accompanies this paper at https://doi.org/10. 1186/s13023-020-01467-9.

\section{Additional file 1. PRISMA 2009 Flow Diagram.}

Additional file 2: Table S1. Clinical trials published for the treatment of EB using small molecules. This table is a summary of published small molecules in the therapy of Epidermolysis bullosa. Publications are ordered by EB subtype and type of trial (RCT, CT, case study), most recent publications on top of each section. The benefit for the patient was clustered into the groups reduction of blister numbers, itch reduction, pain reduction, improvement of wound healing, prevention and treatment of SCCs, and others. Others include QoL $(n=1)$, relieve anal sphincter spasm and fissuring $(n=1)$, lower stricture indices in esophageal stenosis $(n=1)$, plasma phospholipid and fatty acid profiles $(n=1)$, and reduction of inflammation $(n=1)$. na: not applicable. * Primary or clinically relevant endpoints are given for RCT trials. \# Indicates drugs that are currently under clinical investigation in recruiting or running registered trials (Table S2)

Additional file 3: Table S2. Currently registered clinical trials for EB investigation small molecule-based drugs. The table combines trials registered in the following databases: www.clinicaltrials.gov [15] and www. clinicaltrialsregister.eu [16]. Trials are ordered according to current status, subtype and clinical trial phase. Status definitions were adopted from www.clinicaltrials.gov. Recruiting: The study is currently recruiting participants. Active, not recruiting: The study is ongoing, and participants are receiving an intervention or being examined, but potential participants are not currently being recruited. Terminated: The study has stopped early and will not start again. Participants are no longer being examined or treated. Completed: The study has ended normally, and participants are no longer being examined or treated. Unknown: A study whose last known status was recruiting; not yet recruiting; or active, not recruiting but that has passed its completion date, and the status has not been last verified within the past 2 years. Completed registered trials that have subsequently been published are only listed in Table S1

\section{Abbreviations}

EB: Epidermolysis bullosa; EBS: Epidermolysis bullosa simples; EMA: European Medicines Agency; FDA: Food and Drug Administration; JEB: Junctional epidermolysis bullosa; KRT: Keratin; RCT: Randomized controlled trial; RDEB: Recessive-dystrophic epidermolysis bullosa

\section{Acknowledgements}

Not applicable.

\section{Authors' contributions}

W: Conceptualization, writing, data analysis. MR: Research and summary of publications. SK: Research and summary of registered clinical trials. ML: Writing, revision of the final manuscript. The author(s) read and approved the final manuscript.

\section{Funding}

DEBRA Austria.

Availability of data and materials Not applicable.
Ethics approval and consent to participate

Not applicable.

\section{Consent for publication}

Not applicable.

\section{Competing interests}

Verena Wally holds shares of the company Diaderm, which receives license payments from Castle Creek Biosciences (CCB). CCB conducts studies with a diacerein-containing ointment.

Martin Laimer: Grant/Research/Clinical Trial Support: Bristol-Myers Squibb (Squamous cell carcinoma); Onconova Therapeutics; Amryt Pharma; Rehacell; Holostem Terapie Avanzate; Castle Creek Biosciences (Epidermolysis bullosa).

\section{Author details}

${ }^{1}$ EB House Austria, Research Program for Molecular Therapy of Genodermatoses, Department of Dermatology and Allergology, University Hospital of the Paracelsus Medical University, 5020 Salzburg, Austria.

${ }^{2}$ Department of Dermatology and Allergology, University Hospital of the

Paracelsus Medical University Salzburg, Austria, 5020 Salzburg, Austria.

Received: 26 May 2020 Accepted: 21 July 2020

Published online: 19 October 2020

References

1. Has C, Bauer JW, Bodemer C, et al. Consensus reclassification of inherited epidermolysis bullosa and other disorders with skin fragility. Br J Dermatol. 2020. https://doi.org/10.1111/bjd.18921.

2. Uitto J, Bruckner-Tuderman L, McGrath JA, et al. EB2017-Progress in Epidermolysis Bullosa research toward treatment and cure. J Invest Dermatol. 2018;138:1010-6.

3. Castela E, Tulic MK, Rozières A, et al. Epidermolysis bullosa simplex generalized severe induces a $T$ helper 17 response and is improved by apremilast treatment. Br J Dermatol. 2019;180:357-64.

4. Föll MC, Fahrner M, Gretzmeier $C$, et al. Identification of tissue damage, extracellular matrix remodeling and bacterial challenge as common mechanisms associated with high-risk cutaneous squamous cell carcinomas. Matrix Biol. 2018;66:1-21

5. Mittapalli VR, Madl J, Löffek S, et al. Injury-driven stiffening of the dermis expedites skin carcinoma progression. Cancer Res. 2016;76:940-51.

6. Chacón-Solano E, León C, Díaz F, et al. Fibroblast activation and abnormal extracellular matrix remodelling as common hallmarks in three cancerprone genodermatoses. The Br J Dermatol. 2019;181:512-22.

7. Atanasova VS, Russell RJ, Webster TG, et al. Thrombospondin-1 is a major activator of TGF- $\beta$ signaling in recessive dystrophic Epidermolysis Bullosa fibroblasts. J Invest Dermatol. 2019;139:1497-505.

8. Nyström A, Bornert $\mathrm{O}$, Kühl T, et al. Impaired lymphoid extracellular matrix impedes antibacterial immunity in epidermolysis bullosa. PNAS. 2018;115: E705-E14.

9. Annicchiarico G, Morgese MG, Esposito S, et al. Proinflammatory cytokines and Antiskin autoantibodies in patients with inherited Epidermolysis Bullosa. Medicine. 2015;94:e1528

10. Guttmann-Gruber C, Tockner B, Scharler C, et al. Low-dose calcipotriol can elicit wound closure, anti-microbial, and anti-neoplastic effects in epidermolysis bullosa keratinocytes. Sci Rep. 2018:8:13430.

11. Fuentes I, Guttmann-Gruber C, Tay ASL, et al. Reduced microbial diversity is a feature of recessive dystrophic epidermolysis bullosa-involved skin and wounds. J Invest Dermatol. 2018;138:2492-5.

12. Cho RJ, Alexandrov LB, den Breems NY, et al. APOBEC mutation drives earlyonset squamous cell carcinomas in recessive dystrophic epidermolysis bullosa. Sci Transl Med. 2018;10 https://doi.org/10.1126/scitranslmed. aas9668.

13. Pushpakom S, lorio F, Eyers PA, et al. Drug repurposing: progress, challenges and recommendations. Nat Rev Drug Discov. 2019;18:41-58.

14. Hema Sree GNS, Saraswathy GR, Murahari M, et al. An update on drug repurposing: re-written saga of the drug's fate. Biomed Pharmaco Ther. 2019;110:700-16.

15. Clinical trials registry of the FDA. Available at: www.clinicaltrials.gov. Accessed: Apr 2020.

16. Clinical trials registry of the EMA. Available at: www.clinicaltrialsregister.eu. Accessed: Apr 2020. 
17. Reimer A, Lu S, He Y, et al. Combined anti-inflammatory and low-dose antiproliferative therapy for squamous cell carcinomas in recessive dystrophic epidermolysis bullosa. J Eur Acad Dermatol. 2020;34:e1-3.

18. Schrader NHB, Duipmans JC, Molenbuur B, et al. Combined tetrahydrocannabinol and cannabidiol to treat pain in epidermolysis bullosa: a report of three cases. Br J Dermatol. 2019;180:922-4.

19. Pallesen KAU, Lindahl KH, Bygum A. Dominant dystrophic epidermolysis bullosa pruriginosa responding to naltrexone treatment. Acta Derm Venereol. 2019;99:1195-6.

20. Rani S, Gupta A, Bhardwaj M. Epidermolysis bullosa pruriginosa: a rare entity which responded well to thalidomide. Dermatol Ther. 2019;32:e13035.

21. Chelliah MP, Zinn Z, Khuu P, et al. Self-initiated use of topical cannabidiol oil for epidermolysis bullosa. Pediatric Dermatol. 2018;35:224-7.

22. Yaşar Ş, Yaşar B, Cebeci F, et al. Topical sucralfate cream treatment for aplasia cutis congenita with dystrophic epidermolysis bullosa: a case study. J Wound Care. 2018;27:768-71.

23. Chiaverini $\mathrm{C}$, Fontas $\mathrm{E}$, Vabres $\mathrm{P}$, et al. Oral erythromycin therapy in epidermolysis bullosa simplex generalized severe. $\mathrm{Br}$ J Dermatol. 2015;173: 563-4.

24. Leung J, Kuzel P, Kurian A, et al. A case of dominant dystrophic Epidermolysis Bullosa responding well to an old medication. J Am Med Assoc Dermatol. 2015;151:1264-5.

25. Mangold AR, Cole CM, DiCaudo DJ, et al. Treatment of epidermolysis bullosa pruriginosa using systemic and topical agents. J Am Acad Dermatol. 2014;70:e136-7.

26. Ranugha PSS, Mohanan S, Chandrashekar L, et al. Epidermolysis bullosa pruriginosa showing good response to low-dose thalidomide - a report of two cases. Dermatol Ther. 2014;27:60-3.

27. Zanini A, Guez S, Salera S, et al. Oral viscous budesonide as a first-line approach to esophageal stenosis in epidermolysis bullosa: an open-label trial in six children. Paediatr Drugs. 2014;16:391-5.

28. El-Darouti MA, Fawzy MM, Amin IM, et al. Mycophenolate mofetil: a novel immunosuppressant in the treatment of dystrophic epidermolysis bullosa, a randomized controlled trial. J Dermatol Treatment. 2013;24:422-6.

29. Oztekin O, Kalay S, Tezel G, et al. Phenytoin therapy in a neonate diagnosed with epidermolysis bullosa. Int J Dermatol. 2013:52:1376-9.

30. Lara-Corrales I, Parkin PC, Stephens D, et al. The efficacy of trimethoprim in wound healing of patients with epidermolysis bullosa: a feasibility trial. J Am Acad Dermatol. 2012;66:264-70.

31. Dohil R, Aceves SS, Dohil MA. Oral viscous budesonide therapy in children with epidermolysis bullosa and proximal esophageal strictures. J Pediatr Gastroenterol Nutr. 2011:52:776-7.

32. Abitbol RJ, Zhou LH. Treatment of epidermolysis bullosa simplex, weberCockayne type, with botulinum toxin type a. Arch Dermatol. 2009:145:13-5.

33. Younger IR, Priestley GC, Tidman MJ. Aluminum chloride hexahydrate and blistering in epidermolysis bullosa simplex. J Am Acad Dermatol. 1990;23: 930-1.

34. Ozanic Bulic S, Fassihi H, Mellerio JE, et al. Thalidomide in the management of epidermolysis bullosa pruriginosa. Br J Dermatol. 2005;152:1332-4.

35. Banky JP, Sheridan AT, Storer EL, et al. Successful treatment of epidermolysis bullosa pruriginosa with topical tacrolimus. Arch Dermatol. 2004;140:794-6.

36. Fine J-D, Johnson LB, Weiner $M$, et al. Chemoprevention of squamous cell carcinoma in recessive dystrophic epidermolysis bullosa: results of a phase 1 trial of systemic isotretinoin. J Am Acad Dermatol. 2004;50:563-71.

37. Weiner $\mathrm{M}$, Stein $\mathrm{A}$, Cash $\mathrm{S}$, et al. Tetracycline and epidermolysis bullosa simplex: a double-blind, placebo-controlled, crossover randomized clinical trial. Br J Dermatol. 2004;150:613-4.

38. Calikoglu E, Anadolu R. Management of generalized pruritus in dominant dystrophic epidermolysis bullosa using low-dose oral cyclosporin. Acta Derm-Venerol. 2002:82:380-2.

39. Sasidharan CK, Vijayakumar M, Vinodkumor MS. Effect of phenytoin sodium in reducing blistering of epidermolysis bullosa report of four cases. Ind Dermatol, Venereol Leprol. 2002;68:217-9.

40. Marini I, Vecchiet F. Sucralfate: a help during oral management in patients with epidermolysis bullosa. J Periodontol. 2001;72:691-5.

41. Retief CR, Malkinson FD, Pearson RW. Two familial cases of epidermolysis bullosa simplex successfully treated with tetracycline. Arch Dermatol. 1999: 135:997-8.

42. Neufeld-Kaiser W, Sybert VP. Is cyproheptadine effective in the treatment of subjects with epidermolysis bullosa simplex-Dowling-Meara? Arch Dermatol. 1997;133:251-2
43. Hansen SK, Veien NK. Oxytetracycline in epidermolysis bullosa simplex. A double-blind, placebo-controlled trial. J Eur Acad Dermatol. 1996;6:277-8.

44. Takahashi T, Mizutani Y, Ito M, et al. Dystrophic epidermolysis bullosa pruriginosa successfully treated with immunosuppressants. J Dermatol. 2016:43:1391-2.

45. Carroll PB, Rilo HL, Abu Elmagd K, et al. Effect of tacrolimus (FK506) in dystrophic epidermolysis bullosa: rationale and preliminary results. Arch Dermatol. 1994;130:1457-8.

46. Caldwell-Brown D, Stern RS, Lin AN, et al. Lack of efficacy of phenytoin in recessive dystrophic epidermolysis bullosa. Epidermolysis Bullosa study group. N Engl J Med. 1992;327:163-7.

47. White JE. Minocycline for dystrophic epidermolysis bullosa. Lancet. 1989;1: 966.

48. Andreano JM, Tomecki KJ. Epidermolysis bullosa simplex responding to isotretinoin. Arch Dermatol. 1988;124:1445-6.

49. Fine JD, Johnson L. Evaluation of the efficacy of topical bufexamac in epidermolysis bullosa simplex. A double-blind placebo-controlled crossover trial. Arch Dermatol. 1988;124:1669-72.

50. Cunnane SC, Kent ET, McAdoo KR, et al. Abnormalities of plasma and erythrocyte essential fatty acid composition in epidermolysis bullosa: influence of treatment with diphenylhydantoin. J Invest Dermatol. 1987;89: 395-9.

51. Rogers RB, Yancey KB, Allen BS, et al. Phenytoin therapy for junctional epidermolysis bullosa. Arch Dermatol. 1983;119:925-6.

52. Fritsch $\mathrm{P}$, Klein $\mathrm{G}$, Aubock J, et al. Retinoid therapy of recessive dystrophic epidermolysis bullosa. J Am Acad Dermatol. 1983;9:766-7.

53. Bergfeld WF, Orlowski JP. Epidermolysis bullosa letalis and phenytoin. J Am Acad Dermatol. 1982:7.275-8.

54. Bauer EA, Cooper TW, Tucker DR, et al. Phenytoin therapy of recessive dystrophic epidermolysis bullosa. Clinical trial and proposed mechanism of action on collagenase. N Engl J Med. 1980;303:776-8.

55. Wilschanski M, Yahav $Y$, Yaacov $Y$, et al. Gentamicin-induced correction of CFTR function in patients with cystic fibrosis and CFTR stop mutations. $\mathrm{N}$ Engl J Med. 2003;349:1433-41.

56. James PD, Raut S, Rivard GE, et al. Aminoglycoside suppression of nonsense mutations in severe hemophilia. Blood. 2005;106:3043-8.

57. Floquet $C$, Hatin I, Rousset J-P, et al. Statistical analysis of readthrough levels for nonsense mutations in mammalian cells reveals a major determinant of response to gentamicin. PLoS Genet. 2012:8:e1002608.

58. Woodley DT, Cogan J, Hou Y, et al. Gentamicin induces functional type VII collagen in recessive dystrophic epidermolysis bullosa patients. J Clin Invest. 2017;127:3028-38.

59. Li Y, Shen J, Liang J, et al. Gentamicin induces COL17A1 nonsense mutation readthrough in junctional epidermolysis bullosa. J Dermatol. 2020;47:e82e3.

60. Kwong A, Cogan J, Hou Y, et al. Gentamicin induces laminin 332 and improves wound healing in junctional epidermolysis bullosa patients with nonsense mutations. Mol Ther. 2020;28:1327-38.

61. Hammersen J, Neuner A, Wild F, et al. Attenuation of severe generalized junctional epidermolysis bullosa by systemic treatment with gentamicin. Dermatol. 2019;235:315-22.

62. Schwieger-Briel A, Kiritsi D, Schempp C, et al. Betulin-based oleogel to improve wound healing in dystrophic epidermolysis bullosa: a prospective controlled proof-of-concept study. Dermatol Res Practice. 2017;2017: 5068969.

63. Frew Q, Rennekampff HO, Dziewulski P, et al. Betulin wound gel accelerated healing of superficial partial thickness burns: results of a randomized, intraindividually controlled, phase III trial with 12-months follow-up. Burns. 2019; 45:876-90.

64. Krol SK, Kielbus M, Rivero-Muller A, et al. Comprehensive review on betulin as a potent anticancer agent. Biomed Res Int. 2015;2015:584189.

65. Kern JS, Schwieger-Briel A, Löwe $S$, et al. Oleogel-S10 phase 3 study "EASE" for epidermolysis bullosa: study design and rationale. Trials. 2019;20:350

66. Wally $\mathrm{V}$, Lettner $\mathrm{T}$, Peking $\mathrm{P}$, et al. The pathogenetic role of IL-1 $\beta$ in severe epidermolysis bullosa simplex. J Invest Dermatol. 2013;133:1901-3.

67. Mohan GC, Zhang H, Bao L, et al. Diacerein inhibits the pro-atherogenic \& pro-inflammatory effects of IL-1 on human keratinocytes \& endothelial cells. PLOS One. 2017:12:e0173981.

68. Yaron M, Shirazi I, Yaron I. Anti-interleukin-1 effects of diacerein and rhein in human osteoarthritic synovial tissue and cartilage cultures. Osteoarthr Cartil. 1999;7:272-80 
69. Martel-Pelletier J, Pelletier J-P. Effects of diacerein at the molecular leve in the osteoarthritis disease process. Ther Adv Musculoskelet Dis. 2010; 2:95-104.

70. Mendes AF, Caramona MM, de Carvalho AP, et al. Diacerhein and rhein prevent interleukin-1beta-induced nuclear factor-kappaB activation by inhibiting the degradation of inhibitor kappaB-alpha. Pharmacol Toxicol. 2002;91:22-8.

71. Pavelka K, Bruyère $\mathrm{O}$, Cooper $\mathrm{C}$, et al. Diacerein: benefits, risks and place in the management of osteoarthritis. An opinion-based report from the ESCEO. Drugs Aging. 2016;33:75-85.

72. Steinecker-Frohnwieser B, Weigl L, Kullich W, et al. The disease modifying osteoarthritis drug diacerein is able to antagonize pro inflammatory state of chondrocytes under mild mechanical stimuli. Osteoarthr Cartil. 2014;22: 1044-52.

73. Lettner T, Lang R, Klausegger A, et al. MMP-9 and CXCL8/IL-8 are potential therapeutic targets in epidermolysis bullosa simplex. PLoS One. 2013;8:e70123.

74. Wally $\mathrm{V}$, Kitzmueller $\mathrm{S}$, Lagler $\mathrm{F}$, et al. Topical diacerein for epidermolysis bullosa: a randomized controlled pilot study. Orphanet J Rare Dis. 2013;8:69.

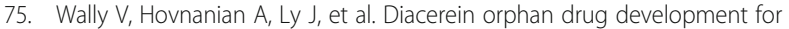
epidermolysis bullosa simplex: A phase 2/3 randomized, placebo-controlled, double-blind clinical trial. J Am Acad Dermatol. 2018:78:892-901 e7.

76. Stander $S$, Yosipovitch $G$. Substance $P$ and neurokinin 1 receptor are new targets for the treatment of chronic pruritus. Br J Dermatol. 2019;181:932-8.

77. Chiou AS, Choi S, Barriga M, et al. Phase 2 trial of a neurokinin-1 receptor antagonist for the treatment of chronic itch in patients with epidermolysis bullosa: a randomized clinical trial. J Am Acad Dermatol. 2019;82:1415-21.

78. Changotade SI-T, Assoumou A, Guéniche F, et al. Epigallocatechin gallate's protective effect against MMP7 in recessive dystrophic epidermolysis bullosa patients. J Invest Dermatol. 2007;127:821-8.

79. Chiaverini C, Roger C, Fontas E, et al. Oral epigallocatechin-3-gallate for treatment of dystrophic epidermolysis bullosa: a multicentre, randomized, crossover, double-blind, placebo-controlled clinical trial. Orphanet J Rare Dis. 2016;11:31.

80. Jain SV, Harris AG, Su JC, et al. The Epidermolysis Bullosa disease activity and scarring index (EBDASI): grading disease severity and assessing responsiveness to clinical change in epidermolysis bullosa. J Eur Acad Dermatol. 2017;31:692-8.

81. Swartling C, Karlqvist M, Hymnelius $K$, et al. Botulinum toxin in the treatment of sweat-worsened foot problems in patients with epidermolysis bullosa simplex and pachyonychia congenita. $\mathrm{Br} \mathrm{J}$ Dermatol. 2010;163:1072-6.

82. Holahan HM, Farah RS, Ferguson NN, et al. Treatment of symptomatic epidermolysis bullosa simplex with botulinum toxin in a pediatric patient. J Am Acad Dermatol Case Rep. 2016;2:259-60.

83. Chaptini $C$, Casey $G$, Harris AG, et al. Botulinum toxin a injection for chronic anal fissures and anal sphincter spasm improves quality of life in recessive dystrophic epidermolysis bullosa. Int J Women's Dermatol. 2015;1:167-9.

84. Ahuja RB, Gupta GK. A four arm, double blind, randomized and placebo controlled study of pregabalin in the management of post-burn pruritus. Burns. 2013;39:24-9.

85. Zou Z, Tao T, Li H, et al. mTOR signaling pathway and mTOR inhibitors in cancer: progress and challenges. Cell Biosci. 2020;10:31.

86. Atanasova VS, Pourreyron C, Farshchian M, et al. Identification of Rigosertib for the treatment of recessive dystrophic Epidermolysis Bullosa-associated squamous cell carcinoma. Clin Cancer Res. 2019;25:3384-91.

87. Kuthiala G, Chaudhary G. Ropivacaine: a review of its pharmacology and clinical use. Indian J Anaesth. 2011:55:104-10.

88. Has C, South AP, Uitto J. Molecular therapeutics in development for Epidermolysis Bullosa: update 2020. Mol Diagn Ther. 2020;24:299-309.

89. Mellerio JE, Uitto J. Meeting Report: The First Global Congress on Epidermolysis Bullosa, EB2020 London - Toward Treatment and Cure, vol. 16; 2020. https://doi.org/10.1016/j.jid.2020.05.078

90. Mavilio F, Pellegrini G, Ferrari S, et al. Correction of junctional epidermolysis bullosa by transplantation of genetically modified epidermal stem cells. Nat Med. 2006;12:1397-402.

91. Hirsch T, Rothoeft $\mathrm{T}$, Teig $\mathrm{N}$, et al. Regeneration of the entire human epidermis using transgenic stem cells. Nature. 2017;551:327-32.

92. Siprashvili Z, Nguyen NT, Gorell ES, et al. Safety and wound outcomes following genetically corrected autologous epidermal grafts in patients with recessive dystrophic epidermolysis bullosa. J Am Med Assoc. 2016; 316:1808-17.
93. Yan WF, Murrell DF. Fibroblast-based cell therapy strategy for recessive dystrophic epidermolysis bullosa. Dermatol Clinics. 2010;28:367-70.

94. Hilgers RD, Bogdan M, Burman CF, et al. Lessons learned from IDeAl - 33 recommendations from the IDeAl-net about design and analysis of small population clinical trials. Orphanet J Rare Dis. 2018;13:77.

95. Unmet needs of EB patients. Available at: http://www.debra-international. org/clinical-guidelines/priorities-for-guidelines.html. Accessed: Oct 2019.

\section{Publisher's Note}

Springer Nature remains neutral with regard to jurisdictional claims in published maps and institutional affiliations.
Ready to submit your research? Choose BMC and benefit from:

- fast, convenient online submission

- thorough peer review by experienced researchers in your field

- rapid publication on acceptance

- support for research data, including large and complex data types

- gold Open Access which fosters wider collaboration and increased citations

- maximum visibility for your research: over $100 \mathrm{M}$ website views per year

At BMC, research is always in progress.

Learn more biomedcentral.com/submissions 